Internat. J. Math. \& Math. Sci.

Vol. 23, No. 1 (2000) 69-76

S0161171200000715

(C) Hindawi Publishing Corp.

\title{
RANDOM TRILINEAR FORMS AND THE SCHUR MULTIPLICATION OF TENSORS
}

\author{
IBRAHIM ALMASRI, JINLU LI, and ANDREW TONGE
}

(Received 27 November 1996 and in revised form 3 March 1998)

\begin{abstract}
We obtain estimates for the distribution of the norm of the random trilinear form $A: \ell_{r}^{M} \times \ell_{p}^{N} \times \ell_{q}^{K} \rightarrow \mathbb{C}$, defined by $A\left(e_{i}, e_{j}, e_{k}\right)=a_{i j k}$, where the $a_{i j k}$ 's are uniformly bounded, independent, mean zero random variables. As an application, we make progress on the problem when $\ell_{r} \breve{\otimes} \ell_{p} \breve{\otimes} \ell_{q}$ is a Banach algebra under the Schur multiplication.
\end{abstract}

Keywords and phrases. Random tensors, Schur multiplication.

2000 Mathematics Subject Classification. 46B09, 46B28, 46A32, 47L05, 47L25.

1. Introduction and notation. We adopt the standard notation $\ell_{p}^{N}(1 \leq p<\infty)$ for the complex vector space $\mathbb{C}^{N}$ equipped with the norm

$$
\|x\|_{p}:=\left(\sum_{n=1}^{N}\left|x_{n}\right|^{p}\right)^{1 / p} .
$$

The usual modifications are made to define $\ell_{\infty}^{N}$ and the infinite dimensional sequence spaces $\ell_{p}(1 \leq p \leq \infty)$. All of these are Banach spaces.

Let $A: \ell_{p}^{N} \rightarrow \ell_{q}^{M}(1 \leq p, q \leq \infty)$ be a linear map and define the operator norm by

$$
\|A\|_{p \rightarrow q}:=\sup \left\{\|A x\|_{q}:\|x\|_{p}=1\right\} .
$$

The map $A$ can be represented as an $M \times N$ matrix $\left(a_{i j}\right)$ with respect to the standard bases. Motivated by problems on absolutely summing operators, Bennett [1] and Bennett, Goodman and Newman [2] obtained estimates for the probability distribution of $\|A\|_{p \rightarrow q}$ when the $a_{i j}$ 's are independent, mean zero random variables bounded by (2.3). They showed that, for all $1 \leq p, q \leq \infty$, the expectation $\mathscr{E}\left(\|A\|_{p \rightarrow q}\right)$ is of the same order as the smallest possible value of $\|A\|_{p \rightarrow q}$ when all the matrix entries $a_{i j}$ are \pm 1 .

Notice that these results can also be interpreted as estimates for the norms of random bilinear forms. Problems involving the von Neumann inequality led Varopoulos [9] to work with norms of random trilinear forms on $\ell_{2}^{N}$. His results were extended and refined by Mantero and Tonge [7]. Let $A: \ell_{p_{1}}^{N} \times \cdots \times \ell_{p_{n}}^{N} \rightarrow \mathbb{C}$ be an $n$-linear form with $A\left(e_{k_{1}}, \ldots, e_{k_{n}}\right)=a_{k_{1}, \ldots, k_{n}}$, where the $e_{k}$ 's are the standard unit basis vectors. There is a natural norm

$$
\|A\|_{p_{1}, \ldots, p_{n}}:=\sup \left\{\left|A\left(x_{1}, \ldots, x_{n}\right)\right|:\left\|x_{i}\right\|_{p_{i}} \leq 1(1 \leq i \leq n)\right\} .
$$

In [7], it was shown that when the $a_{k_{1}, \ldots, k_{n}}$ 's are independent random variables taking the values \pm 1 with equal probability, the expectation $\mathscr{E}\left(\|A\|_{p_{1}, \ldots, p_{n}}\right)$ is of the same 
order as the smallest conceivable value: the least possible value of $\|A\|_{p_{1}, \ldots, p_{n}}$ when each $a_{k_{1} \cdots k_{n}}$ is \pm 1 . These results turned out to be useful in the study of Banach algebra structures on the tensor products $\ell_{p_{1}} \otimes \cdots \otimes \ell_{p_{n}}$. However, open problems were left, even in the case $n=3$.

We address these problems. They involve the Schur product of tensors $A, B \in \ell_{r} \otimes$ $\ell_{p} \otimes \ell_{q}$ when $A=\left(a_{i j k}\right)$ and $B=\left(b_{i j k}\right)$ this is given by

$$
A * B:=\left(a_{i j k} b_{i j k}\right) .
$$

The injective tensor product $\ell_{r} \ddot{\otimes} \ell_{p} \ddot{\otimes} \ell_{q}$ is the completion of $\ell_{r} \otimes \ell_{p} \otimes \ell_{q}$ under the norm

$$
\left\|\left(a_{i j k}\right)\right\|_{r p q}:=\sup \left\{\left|\sum_{i j k} a_{i j k} x_{i} y_{j} z_{k}\right|:\|x\|_{r^{\prime}} \leq 1,\|y\|_{p^{\prime}} \leq 1,\|z\|_{q^{\prime}} \leq 1\right\} .
$$

Here, the index $p^{\prime}$ is the one conjugate to $p$, that is $1 / p+1 / p^{\prime}=1$. Notice that $\|A\|_{r p q}=$ $\|A\|_{r^{\prime}, p^{\prime}, q^{\prime}}$.

In [7], it was shown that the Schur product extends continuously to $\ell_{r} \ddot{\otimes} \ell_{p} \ddot{\otimes} \ell_{q}$ when

(i) the sum of the reciprocals of any two of $p, q$, and $r$ is at least $3 / 2$,

(ii) $1 \leq p, q, r \leq 2$ and the sum of the reciprocals of all three is at least 2 , or

(iii) at least one of $p, q$, and $r$ is 1 or $\infty$.

Cases were also identified where the Schur product did not extend continuously to $\ell_{r} \breve{\otimes} \ell_{p} \breve{\otimes} \ell_{q}$. We extend knowledge of such cases by providing an estimate for the distribution of $\|A\|_{r p q}$ when the $a_{i j k}$ 's are uniformly bounded, independent, mean zero random variables. Our methods build on the techniques of $[1,2,7]$.

Although the problem we consider is relevant to many issues in the geometry of Banach spaces or Banach algebras (see, for example, Diestel, Jarchow, and Tonge [4]), we are not aware of any progress in the last few years. Recent work on the Schur product (see, for example, Horn and Johnson [6]) or on random matrices (see, for example, Girko [5]) mostly focuses on other issues. There is one notable exception, namely, the body of work on completely bounded operators. The basic theory can be found in Paulsen [8], and interesting results closely related to the operator algebra theory, developed by Varopoulos [9] and his group appear in Blecher and Le Merdy [3] and references therein. None of this, however, appears to be directly applicable to the problem we treat in this paper.

2. The probabilistic estimate. We consistently use $\mathscr{P}$ to denote probability and $\mathscr{E}$ to denote mathematical expectation.

Proposition 2.1. Let $1 \leq p \leq 2$ and $2 \leq q, r<\infty$. Let $A=\left(a_{i j k}\right) \in \ell_{r}^{M} \breve{\otimes}_{p}^{N} \breve{\otimes}_{q}^{K}$ and suppose that the $a_{i j k}$ 's are independent, mean zero random variables, and that each $\left|a_{i j k}\right| \leq 1$. Then there are positive constants $C_{1}$ and $C_{2}$, independent of $M, N$, and $K$, such that

$$
\mathscr{P}\left(\|A\|_{r p q}^{r} \geq C_{1} M N^{(r / p)-(r / 2)}+C_{2}(N+K) N^{(r / p)-1} K^{(r / q)-(2 / q)}\right)<1 .
$$


Proof. Our argument is an adaptation of the work in [1, 2]. Note that

$$
\|A\|_{r p q}^{r}=\sup \left\{\sum_{i=1}^{M}\left|\sum_{j, k=1}^{N, K} a_{i j k} y_{j} z_{k}\right|^{r}:\|y\|_{p^{\prime}} \leq 1,\|z\|_{q^{\prime}} \leq 1\right\} .
$$

As in [1] or [2], for any positive $\lambda$ and any nonzero $\left(y_{j}\right)_{j=1}^{N}$ and $\left(z_{k}\right)_{k=1}^{K}$, we have

$$
\mathscr{P}\left(\left|\sum_{j, k=1}^{N, K} a_{i j k} y_{j} z_{k}\right| \geq \lambda\right) \leq 2 \exp \left(-\frac{\lambda^{2}}{4} \sum_{j, k=1}^{N, K} y_{j}^{2} z_{k}^{2}\right) .
$$

If $\mu>0$, then

$$
\begin{aligned}
\mathscr{E}\left(\exp \left(\mu\left|\sum_{j, k=1}^{N, K} a_{i j k} y_{j} z_{k}\right|^{r}\right)\right) & =\int_{0}^{\infty} e^{\mu \lambda^{r}} d \mathscr{P}\left(\left|\sum_{j, k=1}^{N, K} a_{i j k} y_{j} z_{k}\right| \leq \lambda\right) \\
& =1+\int_{0}^{\infty} \mu r \lambda^{r-1} e^{\mu \lambda^{r} \mathscr{P}}\left(\left|\sum_{j, k=1}^{N, K} a_{i j k} y_{j} z_{k}\right|>\lambda\right) d \lambda,
\end{aligned}
$$

and an application of (2.3) gives

$$
\mathscr{E}\left(\exp \left(\mu\left|\sum_{j, k=1}^{N, K} a_{i j k} y_{j} z_{k}\right|^{r}\right)\right) \leq 1+\int_{0}^{N^{1 / p} K^{1 / q}} \mu r \lambda^{r-1} e^{\mu \lambda^{r}} \cdot 2 e^{-\lambda^{2} / 4 \sum_{j, k=1}^{N, K} y_{j}^{2} z_{k}^{2} d \lambda} .
$$

Since $2 \leq r<\infty$, if $0 \leq \mu \leq\left(N^{1 / p} K^{1 / q}\right)^{2-r} / 8 \sum_{j, k=1}^{N, K} y_{j}^{2} z_{k}^{2}$, we can find a constant $C_{1}$, independent of $M, N$, or $K$, such that

$$
\begin{aligned}
\mathscr{E}\left(\exp \left(\mu\left|\sum_{j, k=1}^{N, K} a_{i j k} y_{j} z_{k}\right|^{r}\right)\right) & \leq 1+2 \mu r \int_{0}^{\infty} \lambda^{r-1} e^{-\lambda^{2} / 8 \sum_{j, k=1}^{N, K} y_{j}^{2} z_{k}^{2} d \lambda} \\
& =1+C_{1} \mu\left(\sum_{j, k=1}^{N, K} y_{j}^{2} z_{k}^{2}\right)^{r / 2} .
\end{aligned}
$$

Next, applying independence, we obtain

$$
\begin{aligned}
\mathscr{E}\left(\exp \left(\mu \sum_{i=1}^{M}\left|\sum_{j, k=1}^{N, K} a_{i j k} y_{j} z_{k}\right|^{r}\right)\right) & =\prod_{i=1}^{M} \mathscr{E}\left(\exp \left(\mu\left|\sum_{j, k=1}^{N, K} a_{i j k} y_{j} z_{k}\right|^{r}\right)\right) \\
& \leq \prod_{i=1}^{M}\left(1+C_{1} \mu\left(\sum_{j, k=1}^{N, K} y_{j}^{2} z_{k}^{2}\right)^{r / 2}\right) \\
& \leq \exp \left(C_{1} M \mu\left(\sum_{j, k=1}^{N, K} y_{j}^{2} z_{k}^{2}\right)^{r / 2}\right) .
\end{aligned}
$$

Consequently, for any $v>0$, we have

$$
\mathscr{P}\left(\mu \sum_{i=1}^{M}\left|\sum_{j, k=1}^{N, K} a_{i j k} y_{j} z_{k}\right|^{r} \geq C_{1} M \mu\left(\sum_{j, k=1}^{N, K} y_{j}^{2} z_{k}^{2}\right)^{r / 2}+v\right) \leq e^{-v} .
$$


Now, if $\|y\|_{p^{\prime}} \leq 1$ and $\|z\|_{q^{\prime}} \leq 1$, then since $1 \leq p \leq 2 \leq q<\infty$, we have $\sum_{j, k=1}^{N, K} y_{j}^{2} z_{k}^{2} \leq$ $N^{(2 / p)-1}$. Using the result of the entropy argument in the proof [7, Thm. 1], we get

$$
\mathscr{P}\left(\|a\|_{r p q}^{r} \geq C_{1} M N^{(r / p)-(r / 2)}+\frac{v}{\mu}\right) \leq e^{D(N+K)} e^{-v / 2^{(3 r+1)}},
$$

where $D$ is some positive constant independent of $M, N$, and $K$.

Take $\mu=1 / 8\left(N^{1 / p} K^{1 / q}\right)^{2-r} N^{-(2 / p)+1}$ and $v=2^{3 r+2} D(N+K)$, and set $C_{2}=2^{3 r+5} D$ in (2.9) to get

$$
\mathscr{P}\left(\|A\|_{r p q}^{r} \geq C_{1} M N^{(r / p)-(r / 2)}+C_{2}(N+K)\left(N^{1 / p} K^{1 / q}\right)^{r-2} N^{(2 / p)-1}\right) \leq e^{-D(N+K)} .
$$

Since $e^{-D(N+K)}<1$ for large $N$ and $K$, the result follows.

What we need later is an immediate corollary.

COROLlary 2.2. Let $1 \leq p \leq 2$ and $2 \leq q, r<\infty$. Then there is an $A=\left(a_{i j k}\right) \in$ $\ell_{r}^{M} \breve{\otimes} \ell_{p}^{N} \breve{\otimes} \ell_{q}^{K}$, with each $a_{i j k}= \pm 1$, such that

$$
\|A\|_{r p q}^{2}<C \max \left(M^{2 / r} N^{(2 / p)-1}, N^{2 / p} K^{(2 / q)(1-2 / r)}, N^{(2 / p)-(2 / r)} K^{(2 / r)+(2 / q)(1-2 / r)}\right),
$$

where $C$ is a positive constant independent of $M, N$, and $K$.

The next proposition and its corollary are obtained by making minor adjustments to the arguments above. We present them without proof.

Proposition 2.3. Let $2 \leq p, q, r<\infty$. Let $A=\left(a_{i j k}\right) \in \ell_{r}^{M} \breve{\otimes} \ell_{p}^{N} \breve{\otimes}_{q}^{K}$ and suppose that the $a_{i j k}$ 's are independent, mean zero random variables, and that each $\left|a_{i j k}\right| \leq 1$. Then there are positive constants $C_{1}$ and $C_{2}$, independent of $M, N$ and $K$, such that

$$
\mathscr{P}\left(\|A\|_{r p q}^{r} \geq C_{1} M+C_{2}(N+K) N^{(r / p)-(2 / p)} K^{(r / q)-(2 / q)}\right)<1 .
$$

COROLlaRY 2.4. Let $2 \leq p, q, r<\infty$. Then there is an $A=\left(a_{i j k}\right) \in \ell_{r}^{M} \ddot{\otimes} \ell_{p}^{N} \ddot{\otimes} \ell_{q}^{K}$, with each $a_{i j k}= \pm 1$, such that

$$
\|A\|_{r p q}^{2}<C \max \left(M^{2 / r}, N^{(2 / r)+(2 / p)(1-2 / r)}, K^{(2 / q)(1-2 / r)}, N^{(2 / p)(1-2 / r)} K^{(2 / r)+(2 / q)(1-2 / r)}\right),
$$

where $C$ is a positive constant independent of $M, N$, and $K$.

3. Application to the question of the continuity of Schur multiplication. Now, we turn to the problem left unsolved in Mantero and Tonge [7]: under what circumstances is $\ell_{r} \ddot{\otimes} \ell_{p} \ddot{\otimes} \ell_{q}$ a Banach algebra under Schur multiplication? We give further instances when this is not a Banach algebra. To do this, we use the previous results to show that the following is true for appropriate values of $p, q$, and $r$ :

For each positive $B$, it is possible to find integers $M, N$, and $K$ and an $A=\left(a_{i j k}\right) \in$ $\ell_{r}^{M} \ddot{\otimes} \ell_{p}^{N} \check{\otimes} \ell_{q}^{K}$, with each $a_{i j k}= \pm 1$ for which $\|A * A\|_{r p q}>B\|A\|_{r p q}^{2}$.

For this, it is important to note that, trivially, if $A=\left(a_{i j k}\right) \in \ell_{r}^{M} \breve{\otimes} \ell_{p}^{N} \breve{\otimes}_{q}^{K}$ has each $a_{i j k}= \pm 1$, then

$$
\|A * A\|_{r p q}=M^{1 / r} N^{1 / p} K^{1 / q} .
$$


Proposition 3.1. Let $1<\min (p, q) \leq 2 \leq \max (p, q)<\infty$ and $2 \leq r<\infty$. Then $\ell_{r} \ddot{\otimes} \ell_{p} \ddot{\otimes} \ell_{q}$ is not a Banach algebra under Schur multiplication when

$$
\frac{1}{\min (p, q)}<\frac{2}{r} \cdot \frac{1}{\max (p, q)}+\frac{1}{2}
$$

Proof. We consider the case where $1<p \leq 2 \leq q<\infty$. The other case is similar. Fix $B>0$. By (3.1) and Corollary 2.2, it is enough to show that we can find positive integers $M, N$, and $K$ with

$$
\begin{aligned}
& M^{1 / r} N^{1 / p} K^{1 / q} \\
& \quad>B C \max \left(M^{2 / r} N^{(2 / p)-1}, N^{2 / p} K^{(2 / q)(1-2 / r)}, N^{(2 / p)-(2 / r)} K^{(2 / r)+(2 / q)(1-2 / r)}\right),
\end{aligned}
$$

where $C$ is a fixed positive number, independent of $B, M, N$, or $K$. We can achieve this with $N=K=M^{t}$, where $t>0$ provided that

$$
\begin{aligned}
& M^{t / q}>B C M^{(1 / r)+t((1 / p)-1)}, \\
& M^{1 / r}>B C M^{t((1 / p)+(1 / q)-(4 / q r))} .
\end{aligned}
$$

Inequalities (3.4) hold simultaneously for large $M$ if there is a $t>0$ that satisfies

$$
t\left(\frac{1}{p}+\frac{1}{q}-\frac{4}{q r}\right)<\frac{1}{r}<t\left(\frac{1}{q}-\frac{1}{p}+1\right) .
$$

Such a positive $t$ exists if and only if

$$
\frac{1}{p}<\frac{2}{r} \cdot \frac{1}{q}+\frac{1}{2}
$$

This result is illustrated in Figure 1. Mantero and Tonge [7] showed that, for $2 \leq r<$ $\infty, \ell_{r} \breve{\otimes} \ell_{p} \breve{\otimes} \ell_{q}$ is a Banach algebra under Schur multiplication in the diagonally shaded region, but is not in the horizontally shaded region. Our results assert that $\ell_{r} \ddot{\otimes} \ell_{p} \ddot{\otimes} \ell_{q}$ is not a Banach algebra under Schur multiplication in the heavily shaded region. We use the same shading conventions in all subsequent figures.

If we change the role of the indices $p, q$, and $r$, we obtain the following result which is illustrated and compared to existing knowledge in Figure 2.

Proposition 3.2. Let $1<r \leq 2 \leq p, q<\infty$. Then $\ell_{r} \breve{\otimes} \ell_{p} \ddot{\otimes} \ell_{q}$ is not a Banach algebra under Schur multiplication when

$$
\frac{1}{p} \cdot \frac{1}{q}>\frac{1}{2 r}-\frac{1}{4}
$$

Next, we make use of Corollary 2.4.

Proposition 3.3. Let $2 \leq p, q, r<\infty$. Then $\ell_{r} \breve{\otimes} \ell_{p} \breve{\otimes} \ell_{q}$ is not a Banach algebra under Schur multiplication when

$$
\frac{1}{p}+\frac{1}{q}>\frac{1}{2}, \quad \frac{1}{q}+\frac{1}{r}>\frac{1}{2}, \quad \text { or } \quad \frac{1}{r}+\frac{1}{p}>\frac{1}{2} .
$$




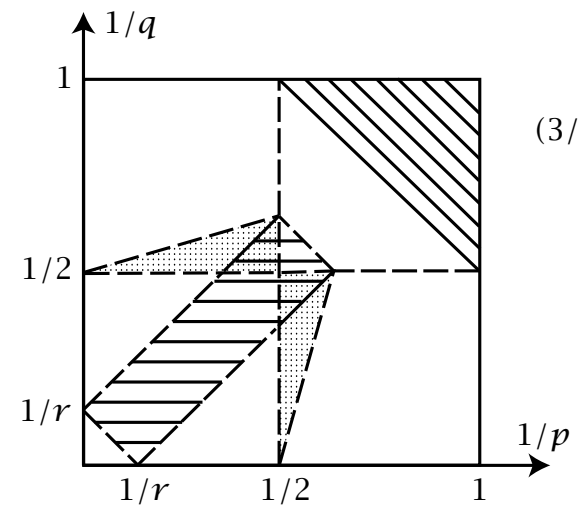

Figure 1

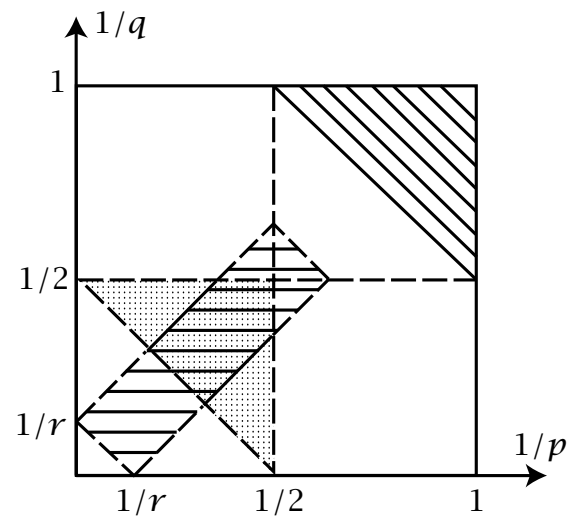

Figure 3

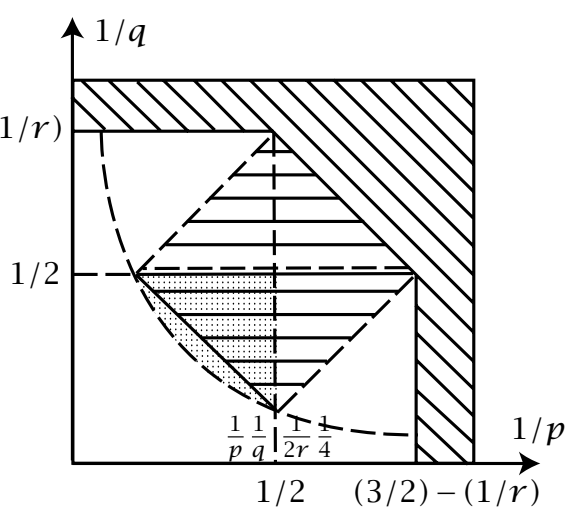

Figure 2

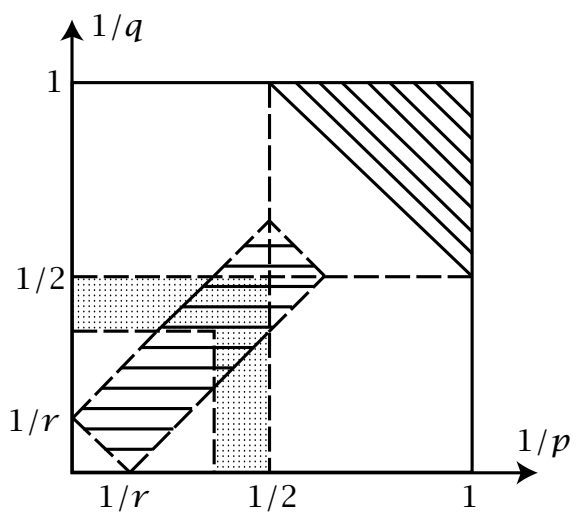

Figure 4

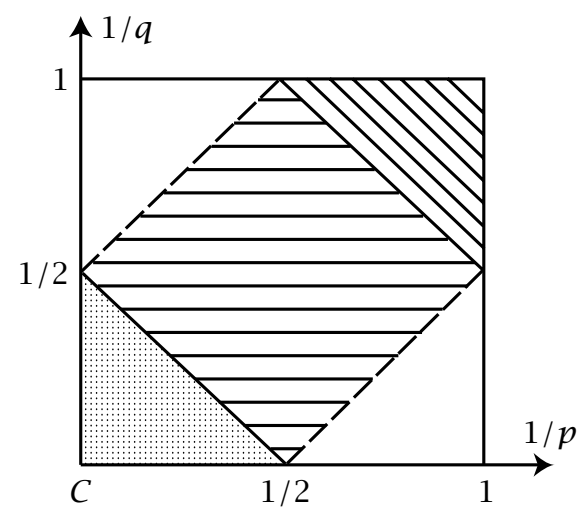

Figure 5 
Proof. Fix $B>0$. By (3.1) and Corollary 2.4, it is enough to show that we can find positive integers $M, N$, and $K$, with

$$
\begin{aligned}
& M^{1 / r} N^{1 / p} K^{1 / q} \\
& \quad>B C \max \left(M^{2 / r}, N^{(2 / r)+(2 / p)(1-2 / r)} K^{(2 / q)(1-2 / r)}, N^{(2 / p)(1-2 / r)} K^{(2 / r)+(2 / q)(1-2 / r)}\right),
\end{aligned}
$$

where $C$ is a fixed positive constant, independent of $B, M, N$, or $K$. We can achieve this with $N=K=M^{t}$, where $t>0$ provided that

$$
\begin{gathered}
M^{t(1 / p+1 / q)}>B C M^{1 / r}, \\
M^{1 / r}>B C M^{(2 t / r)+t(1 / p+1 / q)(1-4 / r) .}
\end{gathered}
$$

Inequalities (3.10) hold simultaneously for large $M$ if there is a $t>0$ that satisfies

$$
t\left(\frac{2}{r}+\left(\frac{1}{p}+\frac{1}{q}\right)\left(1-\frac{4}{r}\right)\right)<\frac{1}{r}<t\left(\frac{1}{p}+\frac{1}{q}\right) .
$$

Such a positive $t$ exists if and only if

$$
\frac{1}{p}+\frac{1}{q}>\frac{1}{2}
$$

The other results follow in a similar manner when the roles of $p, q$, and $r$ are permuted.

The results in Proposition 3.3 are illustrated and compared to previous knowledge in Figures 3 and 4 . The special case when $r=2$ is worth recording separately in Figure 5.

\section{REFERENCES}

[1] G. Bennett, Schur multipliers, Duke Math. J. 44 (1977), no. 3, 603-639. MR 5812490. Zbl 389.47015.

[2] G. Bennett, V. Goodman, and C. M. Newman, Norms of random matrices, Pacific J. Math. 59 (1975), no. 2, 359-365. MR 52 13896. Zbl 325.47018.

[3] D. P. Blecher and C. Le Merdy, On quotients of function algebras and operator algebra structures on $l_{p}$, J. Operator Theory 34 (1995), no. 2, 315-346. MR 96k:46028. Zbl 854.47029.

[4] J. Diestel, H. Jarchow, and A. Tonge, Absolutely Summing Operators, Cambridge Studies in Advanced Mathematics, vol. 43, Cambridge University Press, Cambridge, 1995. MR 96i:46001. Zbl 855.47016.

[5] V. L. Girko, Random matrices, Handbook of algebra (Amsterdam), vol. 1, North-Holland, 1996, pp. 27-78. MR 98m:60052. Zbl 862.15015.

[6] R. A. Horn and C. R. Johnson, Topics in Matrix Analysis, Cambridge University Press, Cambridge, 1994, Corrected reprint of the 1991 original. MR 95c:15001. Zbl 801.15001.

[7] A. M. Mantero and A. M. Tonge, The Schur multiplication in tensor algebras, Studia Math. 68 (1980), no. 1, 1-24. MR 81k:46074. Zbl 445.46051.

[8] V. I. Paulsen, Completely Bounded Maps and Dilations, Pitman Research Notes in Mathematics Series, vol. 146, John Wiley \& Sons, Inc., New York, 1986. MR 88h:46111. Zbl 614.47006 .

[9] N. T. Varopoulos, On an inequality of von Neumann and an application of the metric theory of tensor products to operators theory, J. Funct. Anal. 16 (1974), 83-100. MR 508116. Zbl 288.47006. 
Almasri: College of Engineering AND Technology, Hebron, The West Bank, Palestine

Li: Mathematics Department, Shawnee State University, Portsmouth, OH 45662, USA E-mail address: $\mathrm{j} 1 \mathrm{i} @$ shawnee.edu

Tonge: Department of Mathematics and Computer Science, Kent State University, KENT, OH 44242, USA

E-mail address: tonge@mcs. kent. edu 


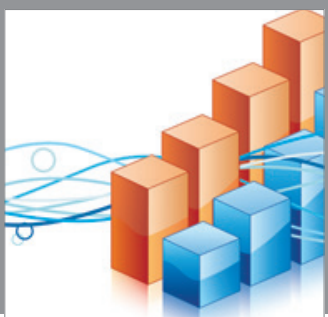

Advances in

Operations Research

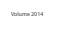

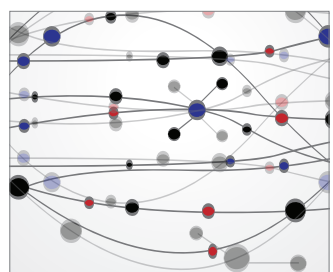

\section{The Scientific} World Journal
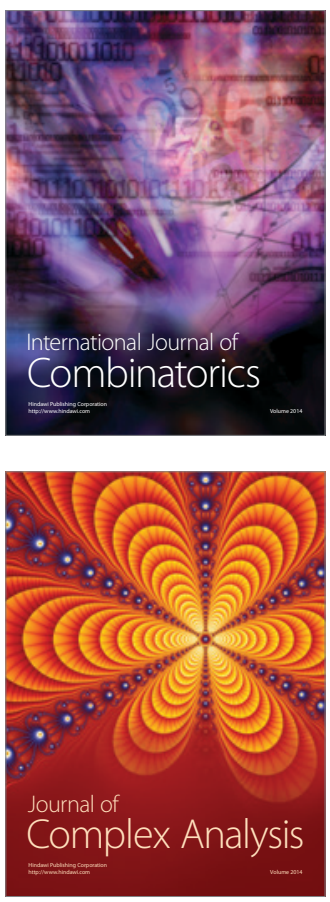

International Journal of

Mathematics and

Mathematical

Sciences
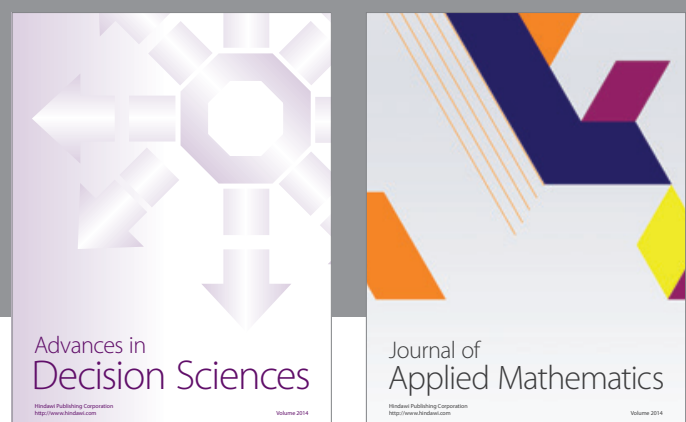

Journal of

Applied Mathematics
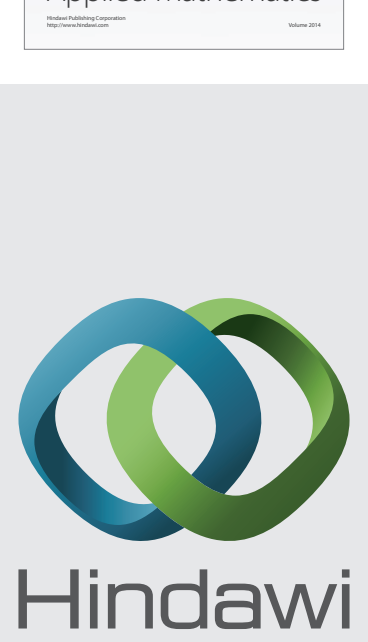

Submit your manuscripts at http://www.hindawi.com
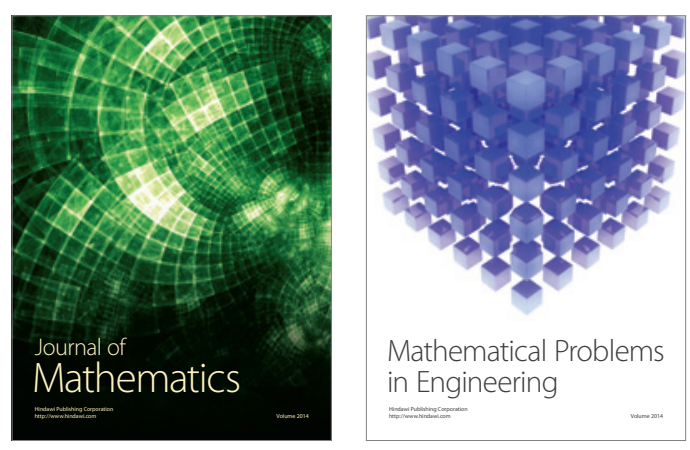

Mathematical Problems in Engineering
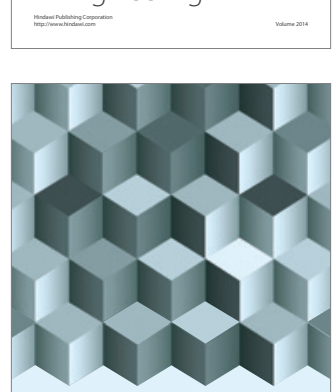

Journal of

Function Spaces
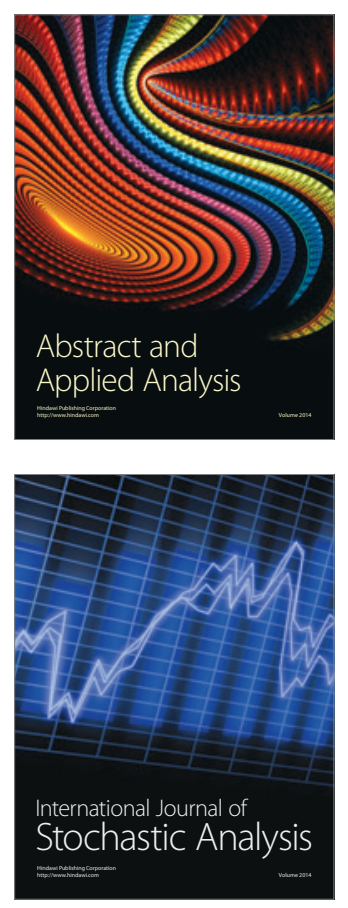



ournal of

Probability and Statistics

Promensencen
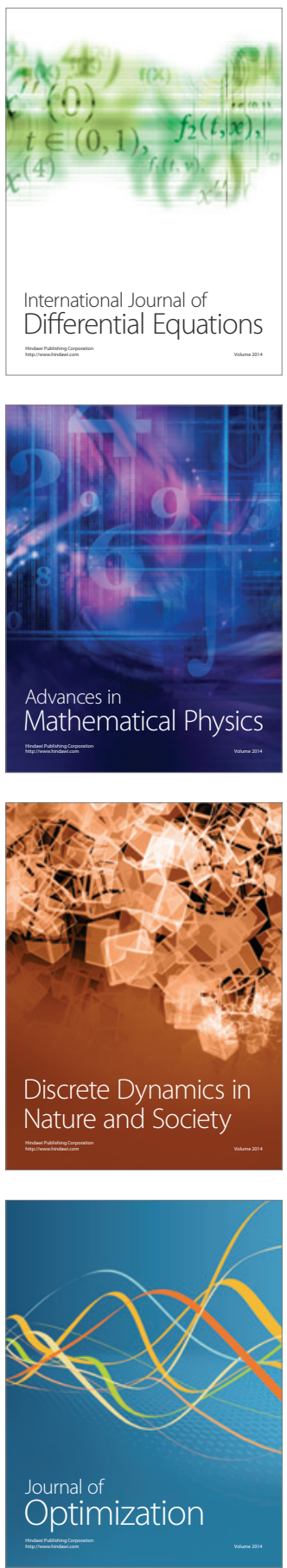